Acquisition et interaction en langue étrangère

5| 1995

L'acquisition de l'italien langue étrangère

\title{
Un aspect du syntagme nominal en italien L2 : le genre
}

Marina Chini

\section{OpenEdition}

1 Journals

Édition électronique

URL : https://journals.openedition.org/aile/4928

DOI : 10.4000/aile.4928

ISSN : 1778-7432

Éditeur

Association Encrages

Édition imprimée

Date de publication : 1 juin 1995

Pagination : 115-142

ISSN : 1243-969X

Référence électronique

Marina Chini, «Un aspect du syntagme nominal en italien L2 : le genre », Acquisition et interaction en

langue étrangère [En ligne], 5 | 1995, mis en ligne le 04 juin 2012, consulté le 14 avril 2022. URL : http:// journals.openedition.org/aile/4928; DOI : https://doi.org/10.4000/aile.4928

Ce document a été généré automatiquement le 14 avril 2022.

(c) Tous droits réservés 


\title{
Un aspect du syntagme nominal en italien L2 : le genre
}

\author{
Marina Chini
}

\section{NOTE DE L'ÉDITEUR}

Je remercie A. Giacalone Ramat pour ses réactions à une première version de ce texte et pour les nombreuses suggestions qu'elle m'a faites pendant la préparation de mon doctorat (Chini 1992a et à paraître), dont cet article présente les principaux résultats. Je remercie aussi pour leurs suggestions deux lecteurs anonymes de la revue.

\section{0 . Introduction}

1 Cet article abordera quelques phénomènes morphosyntaxiques qui ont été observés dans les variétés d'acquisition de l'italien, en particulier dans le secteur de la grammaire du nom et du syntagme qui en est la projection. On s'occupera surtout de la catégorie du genre $(=G)$, sur laquelle on a pu recueillir et étudier les données d'apprenants de différentes langues maternelles (LM) ${ }^{1}$ et les comparer avec celles d'enfants italophones (Chini, 1992a, 1994a) et avec d'autres études concernant le nom dans les interlangues (IL) ${ }^{2}$. Ces réflexions sur l'acquisition du $G$ visent à identifier les principes mis en œuvre et à les confronter avec ceux qui ont été découverts par la linguistique typologique et la psycholinguistique acquisitionnelle de la L1. En particulier on voudrait discuter nos données à la lumière d'importantes études typologiques portant sur la catégorie du $\mathrm{G}$ et sur son accord (cf. Corbett, 1991) et de recherches sur le $G$ dans les variétés enfantines (Mills, 1986 ; Müller, 1990 ; certains travaux dans Slobin, 1985). Il sera intéressant de repérer et discuter les points de convergence et de divergence entre nos interlangues et d'autres systèmes, qu'ils soient en cours d'élaboration, comme dans le cas des enfants apprenant leur LM, ou déjà consolidés, comme pour ce qui est des langues étudiées par la typologie linguistique. 
2 Il nous semble que l'italien se prête à une étude de ce type, car il est doté d'une morphologie plus riche (et pas seulement dans le domaine du verbe, cf. Berretta, 1993) par rapport à d'autres LC, davantage étudiées (français et anglais). Pour ce qui est de l'acquisition du G, c'est là un sujet de recherche particulièrement stimulant car on y retrouve aussi bien des faits formels comme l'accord en $G$, que des faits sémantiques (l'association entre le $G$ et certains champs sémantiques). Il sera intéressant d'étudier jusqu'où la morphologie est véritablement un domaine fragile (Simone, 1988) et peu réceptif à l'interférence de la langue première ou L1 (Berretta, 1990), et de repérer les traces de l'élaboration autonome et créative de l'apprenant visant à identifier les signifiés codifiés dans la grammaire de la LC et les formes de cette codification. Avant d'essayer de répondre à ces questions (en 4), on abordera trois points: une brève description de la catégorie du $G$ en italien (en 1), le traitement de la morphologie dérivative du $G$ et de la catégorisation en $G$ dans les ILs (en 2), l'accord en $G$ dans le syntagme nominal et hors de celui-ci (en 3 ).

\section{La catégorie du genre en italien}

Dans bon nombre de langues, y compris l'italien, le G est une catégorie située entre la syntaxe et la sémantique, un système de classification nominale, au niveau paradigmatique, ayant un volet syntaxique, l'accord. Selon la typologie linguistique récente, l'accord est le facteur décisif pour le G, même s'il y a toujours un noyau sémantique à la base des systèmes de $G$ (Corbett, 1991). En italien (comme en français) cet accord peut être "interne » et concerner les membres variables du syntagme nominal (les déterminants - articles, démonstratifs, possessifs, quantifieurs - et les modificateurs, tels que les adjectifs épithètes), ou « externe » et toucher les pronoms personnels et anaphoriques et les éléments nominaux du prédicat (adjectifs attributs, participes passés après l'auxiliaire essere [être] et, parfois après avere [avoir] $\left.{ }^{3}\right)$. En italien le $\mathrm{G}$ est pertinent pour deux groupes de parties du discours :

a) les noms, « contrôleurs » de l'accord (gender controller, Corbett, 1991 : 151), ceux qui le régissent : ici le $G$ est un fait lexical et en partie sémantique (v. infra) ;

b) les éléments «cibles " accordés avec le nom (articles, adjectifs, etc : gender target Corbett, 1991 : 106-115, 151), pour lesquels le $G$ est un fait formel, syntaxique, sauf dans le cas du pronom, où l'accord en $\mathrm{G}$ peut être sémantique.

En ce qui concerne les noms, la motivation sémantique du G qui leur est attribuée n'est pas toujours claire en italien. Dans un sous-groupe de substantifs désignant des êtres animés le $\mathrm{G}$ grammatical correspond au sexe du référent ${ }^{4}$ (couples hétéronymiques comme padre $\mathrm{m}$. vs. madre $\mathrm{f}$. [père vs. mère], toro $\mathrm{m}$. vs. mucca $\mathrm{f}$. [taureau vs. vache]). L'opposition de sexe peut aussi se traduire en opposition morphologique claire (signore vs. signor-a, dottore vs. dottor-essa, spettatore vs. spetta-trice, Giuseppe vs. Giusepp-ina), ou moins évidente, comme un cantante $m$. vs. una cantante $f$. (chanteur vs. chanteuse) - il y a là une conversion ou dérivation zéro. Il s'agit de la dérivation de $G$, procédé morphologique très répandu et productif qui sert à former des noms $\mathrm{f}$. à partir de noms $\mathrm{m}$. (et, parfois, vice-versa, cf. le néologisme il mammo [le père s'occupant des enfants comme une mère]). Un morphème très commun de ce type est le - $a$ du f. (figlio / figlia, lupo / lupa), qui s'oppose à la terminaison -o, typique du m., et qui coïncide avec le morphème $\mathrm{f}$. des adjectifs et des participes (bella [belle], è caduta [elle est tombée]), tout en étant de nature différente, plutôt dérivative que flexionnelle. Comme elle a une 
valeur sémantique très nette, la dérivation de $G$ pourrait favoriser l'acquisition de la catégorie du G.

Quelques grammairiens signalent des associations tendancielles entre le $\mathrm{G}$ de l'italien et certains champs sémantiques (Serianni, 1988) : l'association entre le m. et les noms de mois, d'arbres, de métaux, de cours d'eau etc. (settembre caldo [septembre chaud], melo fiorito [pommier fleuri], il rame [le cuivre], il Tevere), celle entre le f. et les noms de vertus, de sciences, de fruits, de villes ou nations (la bontà [la bonté], la biologia [la biologie], la mela [la pomme], una Milano dimenticata [un Milan oublié], la Spagna [l'Espagne]). Cependant dans la plupart des cas, surtout pour les noms d'inanimés, la motivation $\mathrm{du} \mathrm{G}$ est formelle et dérive de l'appartenance du nom à une certaine classe flexionnelle. Les classes flexionnelles nominales de l'italien, remontant aux déclinaisons latines, sont :

\begin{tabular}{|l|l|l|l|l|l|l|}
\hline CL. & DECL.LAT & TERM.SG. & TERM.PL. & G & Ex. & Trad.fr. \\
\hline İ̀re & $2 ., 4$ & -0 & $-i$ & m & libro/libri & livre \\
\hline IIème & $1 .$. & $-\mathrm{a}$ & $-\mathrm{e}$ & f. & carta/carte & carte \\
\hline IIIème & 3. & $-\mathrm{e}$ & $-\mathrm{i}$ & m. & cane/cani & chien \\
\hline & & & & f. & ape/api & abeille \\
\hline
\end{tabular}

6 Les deux premières classes sont les plus univoques quant au $\mathrm{G}$, tandis que la troisième, assez importante, pose des problèmes, parce qu'elle comprend des noms des deux $G$ ayant tous les mêmes terminaisons $(-e /-i)$. Il existe d'autres groupes de noms, de dimensions moins considérables, qui sont eux aussi peu transparents quant au G. Les voici :

Tableau 2: Classes flexionnelles secondaires de l'italien

\begin{tabular}{|c|c|c|c|c|c|c|}
\hline CL. & DECL.LAT. & TERM.SG & TERM.PL. & $G$ & Ex. & Trad.fr. \\
\hline \multirow[t]{2}{*}{ IVème } & 3., 5 . & [diverses] & [=sg.] & $\mathrm{m}$. & re & roi \\
\hline & & & & f. & città & ville \\
\hline Vème & 1. ou < grec & $-a$ & $-\mathrm{i}$ & $\mathrm{m}$. & tema/i & thème \\
\hline \multirow[t]{2}{*}{ VIème } & 2., 4. & -o m. & -a f. & m./f. & uovo/uova & œuf \\
\hline & & -o m. & i m./-a f. & m./f. & braccio/i/a & bras \\
\hline VIIème & diverses & -0 & $-\mathrm{i}$ & f. & mano/mani & main \\
\hline
\end{tabular}

7 Le dernier "groupe » est très réduit et contraste donc faiblement avec la règle qui attribue le $\mathrm{G} \mathrm{m}$. aux noms en -o. Les trois autres classes peuvent être source d'erreur, notamment la cinquième (qui comprend des noms $\mathrm{m}$. en - $a$, terminaison typique $\mathrm{du} f$., 
cf. poeta [poète], programma [programme] ) et la sixième, où au pl. le $\mathrm{G}$ du nom, qui est masculin au sg., peut / doit devenir féminin ${ }^{5}$.

D'autres indices phonomorphologiques de $G$ sont les suffixes (productifs ou non) associés à un certain $\mathrm{G}$, et non seulement ceux qui expriment la dérivation de $\mathrm{G}$ (comme -tore m., -essa, -trice f., cf. ci-dessus) : les terminaisons -iere, -one, p.ex., sont associées au $\mathrm{m}$. (salumiere $\mathrm{m}$. [charcutier], cartone $\mathrm{m}$. [carton]), -zione, -tà au f. (respirazione $\mathrm{f}$. [respiration], gioventà f. [jeunesse], cf. Serianni, 1988).

Le $\mathrm{G}$ a aussi un effet morphosyntaxique sur le syntagme nominal de l'italien, qui est étroitement lié à celui de deux autres catégories : le caractère défini / indéfini du syntagme et le nombre, sg. ou pl. (le cas en italien n'a d'importance que pour les pronoms, Berretta, 1993). L'étude détaillée de ces catégories déborderait le cadre de cet article. Nous nous limiterons donc à ce qui concerne le genre. En italien l'accord en nombre est codifié conjointement à celui du $\mathrm{G}$, dans les mêmes contextes (ou sur les mêmes "cibles", cf. ci-dessus), par le moyen de morphèmes discontinus, dont les principaux sont -o pour le m. sg, - $a$ pour le f. sg, -i pour le m. pl., - $e$ pour le f. pl.. D'autre part l'article (défini ou non) est la cible la plus fréquente et souvent le seul indice du $\mathrm{G}$ du nom (cf. il docente $\mathrm{m}$. / la docente f.). La forme non marquée utilisée dans les contextes de neutralisation (de $G$ et de nombre) est normalement celle du m. sg. (qualcuno è uscito [quelqu'un est sorti]). La codification du G sur le pronom comprend différentes formes, toniques (lui m. [il / lui], lei f. [elle]), clitiques objets (lo / la / li / le [le /la / les]), clitiques obliques (gli / le [lui] m. et f.). Dans la langue écrite on trouve aussi egli ou esso m. / ella ou essa f., pl. essi m. / esse f., qui sont parfois remplacés par questo m. / - a f./ - i m. pl. / - ef. pl. dans la langue parlée. Le domaine pronominal, assez complexe, est en voie de restructuration (Berretta, 1993 : 222-234).

Si l'on compare le $G$ de l'italien au $G$ du français, de l'allemand ou de l'anglais, on s'aperçoit qu'en italien celui-ci a un caractère diffus, un aspect syntaxique plus important. En français et en allemand, p.ex., les déterminants pluriels ne signalent pas le $G$ du nom; de plus en français (Mok, 1968; Harris, 1988) l'opposition entre les terminaisons $\mathrm{du} \mathrm{m}$. et du f. est moins saillante que celle de l'italien $(-o /-a)^{6}$. Quant à l'allemand (Eisenberg, 1986), il ne connait pas l'accord des attributs ni des participes passés (cf. italien il ragazzo (è) simpatico vs. la ragazza (è) simpatica, avec l'allemand der Knabe / das Mädchen ist sympatisch- $\varnothing$; Maria è tornata [Marie est revenue] avec Maria ist zurückgekommen-Ø). Finalement en anglais (cf. Corbett, $1991: 169-170 ; 218-222)$ le seul type d'accord en G est celui du pronom (he / she / it ; his / her / its).

11 La catégorie du nombre aussi, plus répandue que celle du $\mathrm{G}$ (elle est la seule qui résiste dans le secteur nominal de l'anglais, cf. town / towns, woman / women), possède en italien une codification plus claire et plus étendue, voire redondante (Simone, 1990 : 167-168). En anglais elle se limite aux noms (étant donné qu'il n'y a pas d'accord en nombre, sauf dans le cas des pronoms: they / them / their). En français parlé elle ne touche qu'un sous-groupe de noms et d'adjectifs (cf. ceux en -al / pl. -aux : animal / animaux, principal / principaux); dans les autres cas, le morphème du pl. -s n'est plus saisissable, sauf en cas de liaison. Dans les syntagmes nominaux ce sont le plus souvent les déterminants, articles, démonstratifs ou possessifs, qui permettent de distinguer le sg. du pl. (des / les / ces / mes livres chers; Mok, 1968). Voici un exemple :

Le mie amiche sono tornate da Parigi. 


\begin{tabular}{|l|}
\hline f.pl.f.pl. f.pl. $\quad$ f.pl. \\
\hline Le ho viste soddisfatte. \\
\hline f.pl. f.pl. f.pl. \\
\hline Fr.: $\quad$ Mes amies sont revenues de Paris. \\
\hline pl. (f.pl.) $\quad$ (f.pl.) \\
\hline Je les ai vues satisfaites. \\
\hline pl. (f.pl.) f.(pl.) \\
\hline
\end{tabular}

12 Ce caractère diffus de la morphologie nominale flexionnelle de l'italien devrait aider l'apprenant et le guider dans sa découverte de la grammaire de la LC. Cependant la tâche acquisitionnelle pourrait être compliquée par le fait que la plupart des morphèmes portant le $\mathrm{G}$ (et le nombre) sont atones ou clitiques (articles), qu'il y a des irrégularités, des allomorphismes et des homophonies (le est un article défini $\mathrm{f}$. pl. : le case [les maisons], un clitique objet de troisième personne du pl. f. : non le vedo [je ne les f. pl. vois pas], un clitique datif f. sg. : io le chiedo [Je lui demande]). Plusieurs marques codifient le même signifié grammatical (p.ex. pour le f. pl. on a soit -e, penn-e nuov-e [des plumes neuves] soit - $i$, attric-i celebr- $i$ [des actrices célèbres], soit - $a$, uova [œufs]), ce qui contraste avec le principe acquisitionnel « une forme-une signification », si important tant pour L1 que pour L2 (Slobin, 1985 ; Andersen, 1984a).

\section{Le nom dans les interlangues}

13 2.0. Notre étude se fonde sur un corpus concernant treize apprenants de quatre LMs différentes (persan, allemand, français, anglais) ${ }^{7}$. Ce sont des langues indoeuropéennes ayant un système de $G$ différent sur un ou sur plusieurs points de celui de l'italien, le persan étant complètement dépourvu de cette catégorie morphologique (Windfuhr, 1979). Les sujets étudiés sont les membres de deux familles persanes, A et B, immigrées d'Iran pour des raisons socio-politiques en 1988 (Tableaux 3 et 4), et cinq autres apprenants, âgés de 20 à 30 ans (Tableau 5), immigrés pour des raisons d'étude et/ou de travail et pour une période limitée (sauf dans le cas d'Henri). Tous habitaient depuis quelques mois dans le Canton suisse italophone du Tessin (détails dans Chini, 1992a, $1992 b$ et à paraître) et ont été suivis longitudinalement sur une période de durée variable :

Tableau 3 : Famille $\mathrm{A}(\mathrm{L} 1=$ persan$)$

\begin{tabular}{|l|l|l|l|}
\hline MEMBRE & SIGLE & AGE & ENREGISTREMENTS APRES X MOIS DE SEJOUR \\
\hline père & PA & 44 & $5 ; 6 ; 12 ; 13 ; 14$ \\
\hline mère & MA & 35 & $5 ; 6 ; 10 ; 11 ; 12 ; 13 ; 14 ; 15 ; 16$ (bref) \\
\hline
\end{tabular}




\begin{tabular}{|l|l|l|l|}
\hline fils & F1A & 13 & $5 ; 6 ; 10 ; 11 ; 12 ; 13 ; 14 ; 15 ; 16 ; 17 ; 18$ \\
\hline fille & F2A & 10 & $5 ; 6 ; 10 ; 11 ; 12 ; 13 ; 14 ; 15 ; 17 ; 18$ \\
\hline
\end{tabular}

Tableau 4 : Famille B (L1=persan)

\begin{tabular}{|l|l|l|l|}
\hline MEMBRE & SIGLE & AGE & ENREGISTREMENTS APRES X MOIS DE SEJOUR \\
\hline père & PB & ca40 & $7 ; 9 ; 12 ; 14 ; 19$ (bref) \\
\hline mère & MB & ca25 & $6 ; 7 ; 9 ; 12 ; 14 ; 15 ; 16 ; 19$ \\
\hline I fils & F1B & 11 & $6 ; 9 ; 12 ; 14 ; 15 ; 19$ \\
\hline II fils & F2B & 10 & $9 ; 12 ; 14 ; 15 ; 19$ \\
\hline
\end{tabular}

Tableau 5 : Autres apprenants

\begin{tabular}{|l|l|l|l|}
\hline NOM & L1 & AGE & ENREGISTREMENTS APRES X MOIS DE SEJOUR \\
\hline Ava & anglais & 19 & $3 ; 4 ; 6 ; 7$ (bref); 9 \\
\hline Edgar & anglais & 17 & $12 ; 14 ; 16$ \\
\hline Anna & allemand & 19 & $3 ; 4 ; 5 ; 6 ; 7$ \\
\hline Sarah & allemand & 18 & $2 ; 3 ; 5 ; 6$ \\
\hline Henri & français & 28 & $12 ; 15 ; 16 ; 18 ; 19 ; 20 ; 22 ; 26$ \\
\hline
\end{tabular}

14 Il ne nous est possible ici que de résumer l'évolution générale des interlangues examinées (là où elle a lieu); pour plus de détails sur les parcours individuels des apprenants et sur leur LM, cf. Chini 1992a, 1992b et à paraître.

\subsection{Classification et flexion}

15 On explorera davantage le secteur du nom, contrôleur du genre, dont dépend l'accord. Pendant les premières phases, plutôt pragmatiques, des ILs étudiées, les noms fonctionnent comme des signes morphologiquement inanalysés, car ils ne sont utilisés que pour leur valeur lexicale. Bien qu'on puisse y rencontrer des terminaisons de nombre (et de G), claires du point de vue de la LC, il est peu probable qu'elles soient interprétées comme telles. Très souvent les apprenants respectent la terminaison que le mot (sg.) a dans la LC ${ }^{8}$ et posent ainsi les fondements du classement (formel) des noms et donc de la catégorisation en $\mathrm{G}$ (cf. § 1.).

16 Chez des apprenants de LM différente, on observe parfois une certaine tendance à surutiliser la terminaison $-a$ dans la morphologie nominale, même lorsque cela 
contredit le G grammatical et/ou naturel : cf. uoma pour uomo (homme) (MA), une filma pour un film (PB), gusta pour gusto (goût, Sarah, après $5 \mathrm{mo}$.), un globa, sulla corpa, (Edgar, 14 mo. ; Chini, 1992a, 1994a). On a expliqué cette tendance pidginisante en supposant que la terminaison - $a$ fonctionne comme une voyelle typiquement italienne, plus probable, moins marquée au niveau phonologique (Chini, 1992b, 1994a ; Berretta, 1990 : 193).

Lorsque l'analyse morphologique est commencée, surtout quand il s'agit de noms m. ou f. peu typiques, qui n'appartiennent pas à la première ou à la deuxième classe (cf. Tableau 1), les apprenants régularisent les désinences dans la direction des terminaisons typiques du m. (-o) ou du f. (-a) : bicchiero pour bicchiere m. (verre, Henri, $19 \mathrm{mo}$.) ; il problemo pour il problema m. (le problème, Henri $26 \mathrm{mo}$.) ; nomo pour nome m. (nom), coloro pour colore m. (couleur, PA) (Chini, 1992a, 1992b: 455 , 1994a; cf. Valentini, 1990).

Parfois l'apprenant, généralement français et plus rarement germanophone, non seulement intervient sur la terminaison, mais choisit aussi une base lexicale tirée de la LM, inexistante en italien ou de signifié différent. Pendant les premiers mois, il y ajoute la terminaison "typiquement italienne»-a: paisa (fr. pays) pour paese, Ken (francophone du Zaïre, 3 et 7 mo.), leguma (fr. légumes) pour verdure, tomata (fr. tomates) pour pomodori, (Ken) ; parfois il emploie la terminaison de la LC qui correspond au G du mot source dans la LM (cf. una tampeta, Henri 15 mo. pour una tempesta f., du fr. tempête f. ; sul turmo [sur la tour], au lieu de sulla torre f., de l'allemand Turm m., chez Sarah, 5 mo.). On voit là un procédé créatif ayant ses racines dans une «hypothèse générale de construction de lexèmes", proposée par Giacobbe (1989) pour le français LC d'hispanophones et appliquée par des apprenants qui jugent la LM et la LC suffisamment proches. Cette stratégie révèle non seulement le recours "passif » à la LM, mais montre aussi la mise en place d'une compétence phonomorphologique importante de la LC, celle qui suggère le choix de terminaisons italiennes cohérentes avec le $\mathrm{G}$ (réputé) correct.

D'après nos données, les critères d'attribution du $G$ aux noms semblent être d'abord phonologiques (terminaisons), puis sémantiques (sexe du référent) ou morphologiques (suffixes). La force des critères phonologiques est claire aussi dans le cas d'accords tels que una problema (Henri $18 \mathrm{mo}$.) pour un problema (m. mais avec une terminaison typique du f.), où le critère phonologique s'impose même sur le $G$ du nom correspondant de la LM (problème est $\mathrm{m}$. en français). Ces accords "par assonance " rappellent le surface rhyming phenomenon proposé par Levy (1983) pour l'hébreu L1 et qui émerge parfois aussi chez des enfants italophones, en contradiction avec le $G$ naturel (ou sexe du référent) : la Danluca pour (il) Gianluca m. (Jean-Luc), produit par une fille de 2 ans et 10 mois (Chini, 1994a : 414).

Le G que le nom a dans la LM ne paraît influencer l'attribution du G dans la LC que dans un nombre limité de cas, p.ex., les emprunts adaptés (ils sont assez rares, cf. turmo cidessus) ou quelques noms pour lesquels les critères de $G$ de l'italien ne sont pas univoques (noms en -e : la mare, Henri 15 mo., 20 mo., pour il mare m. [la mer] ; la stessa colore, Henri 18 mo., pour lo stesso colore m. [la même couleur]). Parfois la désinence est changée conformément au $\mathrm{G}$ choisi (limona f., Sarah, cf. allemand Zitrone f., au lieu de limone m.; polvero m. pour polvere f., cf. allemand Pulver neutre, Staub m., Puder m.). Rares sont les cas où l'on change les terminaisons nominales de -o en - $a$ ou vice versa sous l'influence du G du nom correspondant dans la LM : lavora pour lavoro m. (travail), 
Sarah 2 mo., cf. allemand Arbeit f.; un piccolo piazzo pour una piccola piazza (une petite place, Sarah 6 mo., cf. allemand Platz m.) (Chini, 1992a, 1994a).

21 Après une période où les seules marques flexionnelles sur le nom sont des pl. inanalysés (souvent des cas d'inversion du principe de « marque » : tre mesi, $\mathrm{MB}, 6 \mathrm{mo}$; dieci anni, F1B, 6 mo. ; tutti persone, PB 7 mo., cf. Chini, 1992b, 1994b), quand la flexion en nombre commence à s'élaborer dans l'IL, la plupart des apprenants, ayant différentes $\mathrm{LM}$, tendent à généraliser l'emploi d'un seul morphème pour le pl., selon le principe " une forme (ici un morphème) - une signification (pl.) », au détriment de l'opposition de G. C'est souvent la forme la plus univoque, celle du m. pl. -i (cf. Tableaux 1 et 2), qu'ils choisissent: $i$ scuoli pour le scuole (les écoles), dei personi pour delle persone (des gens, Edgar, 12 mo.), i donni pour le donne (les femmes, Anna, 4 mo., Chini, 1992a, 1994b ; cf. les sinophones de Valentini, 1990). Chez des francophones et des germanophones il est aussi possible de trouver au pl. la terminaison -e (qui serait f.), surtout pour des noms de la troisième classe en -e (tre mese, pour mesi [trois mois], Henri 12 mo., le sue nonne pour $i$ suoi nonni [ses grands-parents] Henri $18 \mathrm{mo}$; molte restorante pour molti ristoranti [beaucoup de restaurants], Sarah 6 mo. ; cf. Chini, 1994b).

\subsection{Dérivation de $\mathrm{G}$}

La dérivation de $G$ occupe un niveau intermédiaire entre la formation des mots (elle produit des mots nouveaux) et la flexion (elle se sert souvent de moyens semblables aux terminaisons flexionnelles, cf. le - $a$ de ragazz- $a$ et simpatic- $a$ ). Par le moyen d'élicitations orales (pour lesquelles il s'agissait de former des noms f. à partir de noms m.: ex. studentessa [étudiante] à partir de studente [étudiant]), on a pu mettre en évidence une hiérarchie des morphèmes utilisés, qui paraît valable pour tous nos apprenants (Chini, 1992a, 1992b, 1994a) :

$$
\text { - } \mathrm{a}>\text {-essa }>\text { (pseudo-)diminutifs > -trice }
$$

23 Cette hiérarchie reflète la productivité, la fréquence et la transparence des mêmes moyens morphologiques dans la LC : les marques auxquelles on a recours en premier et le plus souvent sont à gauche de la séquence, d'abord- $a$ (cf. aussi les sinophones de Valentini, 1990 : 342), mais aussi, plus tard, -essa, plus saillant et plus lourd. La marque située à droite, -trice, typique des noms d'agent mais plus rare, semble être évitée (cf. direttoressa pour direttrice, Henri, 20 mo. ; F2A, 17 mo. ; Ava, 9 mo., ou direttora, F1A, 17 mo.), ou éventuellement reformulée d'une façon plus transparente : imperatorice, pour imperatrice, à partir de imperatore, chez F2B, 19 mo.) ${ }^{9}$. Quant aux hétéronymes (padre / madre, fratello / sorella, etc.) on les trouve chez beaucoup d'apprenants dès les premiers enregistrements (Chini, 1992b : 457 ; cf. Valentini, 1990 : 342 pour des chinois). Il s'agit de mots appartenant au vocabulaire de base et dont l'apprentissage (y compris celui du G) est un fait purement lexical.

La dérivation de $G$ semble suivre les mêmes principes de "morphologie naturelle " (Dressler et al., 1987) que la dérivation en général, dans l'acquisition ${ }^{10}$ et dans les variétés adultes. On observe avant tout la recherche d'une transparence morphosémantique et morphotaxique maximale, qui peut induire l'apprenant à ajouter, plutôt qu'à supprimer du matériel morphologique (cf. atlet-essa pour atleta), à substituer à des morphèmes opaques des morphèmes plus transparents (imperatorice pour imperatrice) ou plus fréquents (imperatoressa) et à régulariser la base lexicale et/ou le morphème dérivatif (canessa de cane, pour cagna, avec base allomorphe; cf. buonità 
[bonté] pour bontà, de buono [bon], Berretta, 1987 : 43). Les morphèmes dérivatifs les plus productifs $(-a,-e s s a)$ sont généralisés; la dérivation zéro (ou conversion, cf. rinunciare > rinuncia) est peu fréquente tant pour le $\mathrm{G}$ (la leone, la infermiere, anglophone Ava 9 mo.) qu'en général (Berretta, 1987 ; Bozzone Costa, 1986). Ce procédé, peu naturel et peu iconique (Dressler et al., 1987), qui enfreint la correspondance biunivoque entre signifié et signifiant, est appliqué surtout par les anglophones (leur LM l'utilise beaucoup) et par les apprenants débutants, n'ayant pas encore appris les différents morphèmes dérivatifs.

En général les choix morphologiques concernant le $\mathrm{G}$ du nom sont conformes aux tendances $\mathrm{du}$ système de la LC et témoignent une remarquable sensibilité morpholexicale. La LM joue par contre un rôle assez réduit au niveau des formes; elle agit plutôt au niveau des propriétés structurelles, en favorisant l'attention aux désinences ou à un certain type de dispositifs morphologiques (cf. la conversion chez les anglophones).

\section{L'accord en genre}

Venons-en au côté morphosyntaxique et formel de l'acquisition du G: l'accord. Au cours des premières phases, qui peuvent se prolonger longtemps (surtout dans le cas d'apprenants isolés ou ayant une LM très différente de l'italien - persan, chinois, p. ex.), la notion d'accord n'est probablement pas encore à l'œuvre. Les adjectifs et les participes sont souvent inanalysés et les articles peuvent être presque ou totalement absents, comme dans les pidgins. Les signifiés que la LC codifie morphologiquement (nombre, genre, caractère défini ou indéfini, cf. § 1) sont alors souvent laissés implicites, ou bien confiés soit au contexte, à la communication non-linguistique soit aux connaissances du monde. Voici un exemple, tiré d'un récit d'un persan, F1A, de 13 ans, immigré au Tessin depuis 18 mois.

F1A : $\begin{aligned} & \text { ho visto 0i lunapark + dopo+ dentro od lunapark c'è (il) circo + dopo andato dentro + } \\ & \text { comperato 0d/i bi/ bilietto [...] dopo tanti gente + con quello + eh c'è 0i bicicletta che con una } \\ & \text { ruota + dopo andavano su quello bicicletta + dopo fanno una aereo }{ }^{11}\end{aligned}$

Si le niveau verbal est plutôt simple, mais de toute façon dynamique (cf. les participes passés, les passés composés et les imparfaits), la morphosyntaxe nominale se révèle très réduite $: \mathrm{il} \mathrm{y}$ a des omissions de morphèmes semi-libres tels que des articles définis (marquées $0 \mathrm{~d}$ ) et parfois indéfinis (0i), tandis que des adjectifs prénominaux (des démonstratifs et un quantifieur) ne sont pas (correctement) accordés ${ }^{12}$.

Plus généralement, en étudiant tous les contextes d'accord (effectué ou manqué) dans les données des apprenants étudiés (\$ 2.0.), on a pu proposer une séquence acquisitionnelle de l'accord dans le secteur nominal des ILs qui est la suivante (Chini, 1992a, 1992b, 1994a et b) :

Pronom pers. 3ème pers. sg. > art. déf. (>) art. indéf. > adj. épithète > adj. attribut (>) participe passé ${ }^{13}$

29 Tous les apprenants utilisent presque dès le début les pronoms toniques fléchis de la troisième personne du sg. (lui $\mathrm{m}$. et lei f.), avec très peu de fautes ${ }^{14}$. Il s'agit d'un accord important au niveau sémantique, ce qui pourrait expliquer cette priorité. Pour le reste 
la probabilité de l'accord diminue à mesure qu'augmente la distance entre le contrôleur (nom) et sa cible, distance qui est maximale dans le cas de l'attribut et du participe passé, plus petite dans le cas de l'article ou de l'adjectif du syntagme nominal. Voilà un premier résultat intéressant, qui peut être mis en relation avec des généralisations de la recherche typologique ( $\$ 4$, point II). Mais voyons plus en détail les différents domaines de l'accord.

Pour ce qui est des articles, ils sont d'abord souvent omis (les adultes persanophones les omettent presque toujours : F1A 90\% des cas), puis on les accorde surtout s'ils sont définis. La sémantique du nom, c'est-à-dire le fait d'avoir un référent sexué, n'est pas un facteur susceptible de déclencher l'accord de l'article; la terminaison - $a$ du nom est par contre plus importante pour déclencher l'accord au féminin. Les formes attestées d'articles sont : la, il sg. ; le, i pl. ; un / uno / una ; les allomorphes lo et gli apparaissent beaucoup plus tard. L'élision des articles semble être évitée afin de garder leur transparence (una alternativa molto interessanta [une alternative très intéressante], Henri 20 mo.; cf. aussi Berretta, 1990: 194-195 et Berruto et al., 1990). Certains groupes d'apprenants, notamment les persanophones adultes, réduisent énormément le système des articles: ils emploient seulement una / une pour l'indéfini et, très rarement, la comme défini (Chini, 1992b) ${ }^{15}$. Le f. la est préféré et parfois généralisé aussi par d'autres apprenants (Chini, 1992a, 1994a ; cf. Berretta, 1990 ; Berruto et al., 1990 ; Valentini, 1990), sauf par les anglophones, qui choisissent $i l$, même dans le cas de noms typiquement f. (il donna, il moglie, Ava 4 mo.; il guerra, John 2 mo.; cf. aussi il chiave, Edgar 16 mo.; Chini, 1992a). Au pluriel beaucoup préfèrent l'article le (le sue genitore pour i suoi genitori [ses parents], Henri 18 mo. ; le libri pour i libri [les livres], F1B 12 mo.; le piatti pour $i$ piatti [les assiettes], Anna $7 \mathrm{mo}$.), et pas seulement les francophones qui pourraient s'appuyer sur la forme les de leur LM : l'article le a un corps phonique plus consistant que le $\mathrm{m}$. $i$ et il est plus cohérent avec le système des articles définis, formés sur le modèle : latérale + morphème de $G$ et de nombre.

31 En général l'article indéfini est utilisé plus tôt que l'article défini, mais il est accordé plus tard, peut-être parce que sa fonction textuelle (et son identification avec le numéral, normalement invariable en italien) est plus saillante que l'aspect morphosyntaxique ou bien parce qu'il occupe une position syntaxique profonde plus éloignée du nom qui régit l'accord (Chomsky, 1981). La forme la plus fréquente et généralisée est le $\mathrm{m}$. un, tandis que, pendant les premières phases, quelques apprenants peu avancés, persanophones et germanophones, préfèrent le (pseudo-)f. una (une), à désinence vocalique (comme la ; cf. aussi Berruto et al., 1990).

S'il est vrai que des morphèmes semilibres comme les articles peuvent être omis assez facilement, il ne saurait en être de même pour des morphèmes liés (désinences adjectivales et participiales): dans ce domaine les ILs tendent à utiliser des terminaisons non marquées, typiquement celles du m. sg. (Chini, 1992a, 1992b; cf. Berretta, 1990, Valentini, 1990) et l'absence d'accord est alors plus prolongée que pour les articles. Quant à l'accord interne au syntagme, il semble que, lorsque les adjectifs occupent une position prénominale (démonstratifs, indéfinis, possessifs), ils sont accordés avant les épithètes postnominaux (cf. Valentini, 1990: 342, pour un sinophone, et Berretta \& Crotta, 1991), mais cela ne ressort pas toujours clairement de nos données ${ }^{16}$. L'attribut reste sans doute longtemps non marqué, au m. sg. : io sono allergico, pour allergica f. (Ava, 6 mo.), la cucina è piccolo (Anna, 7 mo.), Siena è troppo bello 
(Henri, 20 mo.). A cause de sa nature verbale le participe résiste à l'accord plus que l'attribut (io sono stato [j'ai été] pour io sono stata, Ava, 9 mo.).

33 Avant de discuter ces résultats, nous présentons à titre d'exemple quelques graphiques et deux tableaux. Les premiers visualisent le choix préférentiel et prévalent pour les marques d'accord qui sont $\mathrm{m}$. selon la LC (Graphiques 1, 2 et 3).

Graphique 1 : Marques de $\mathrm{G}$ sur le total des cibles pour MB

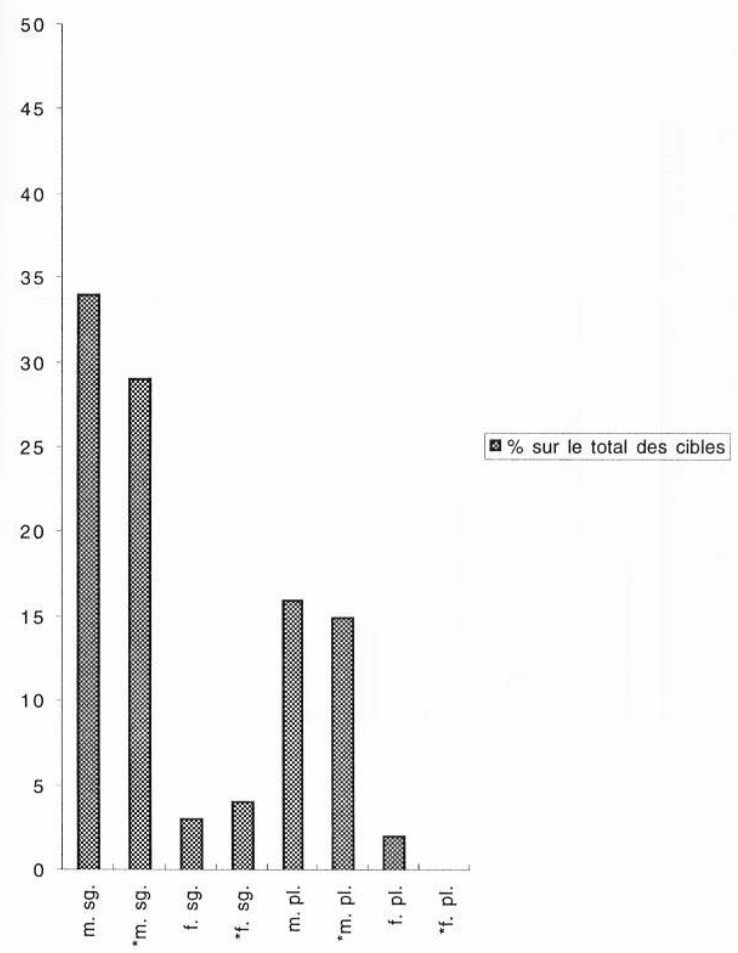


Graphique 2 : Marques de $\mathrm{G}$ sur le total des cibles pour F1B

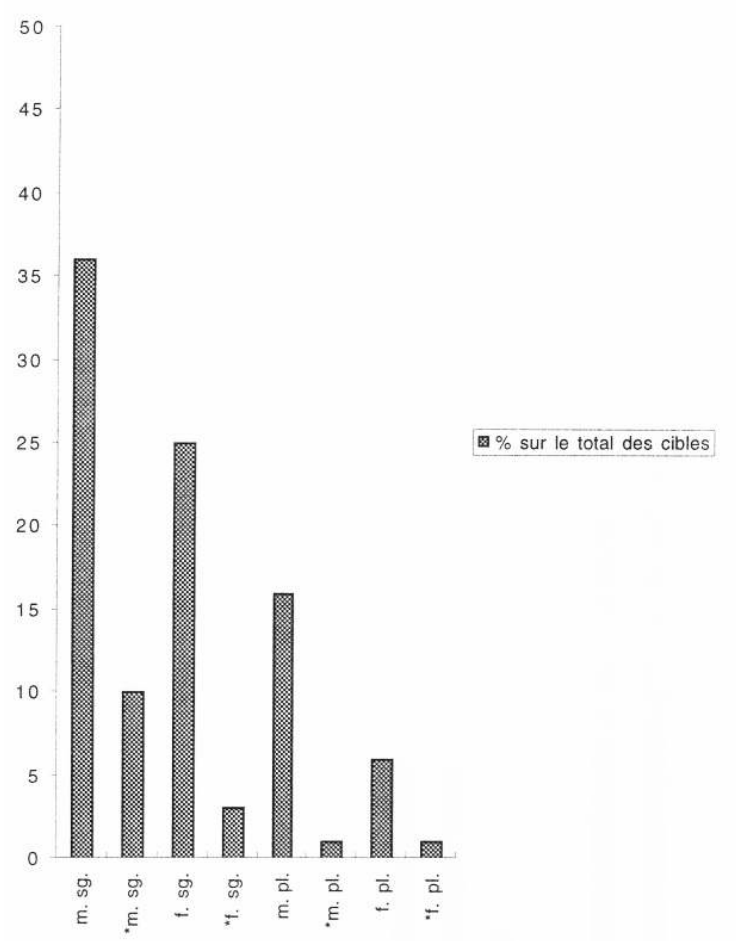

Graphique 3 : Marques de G sur le total des cibles pour Anna

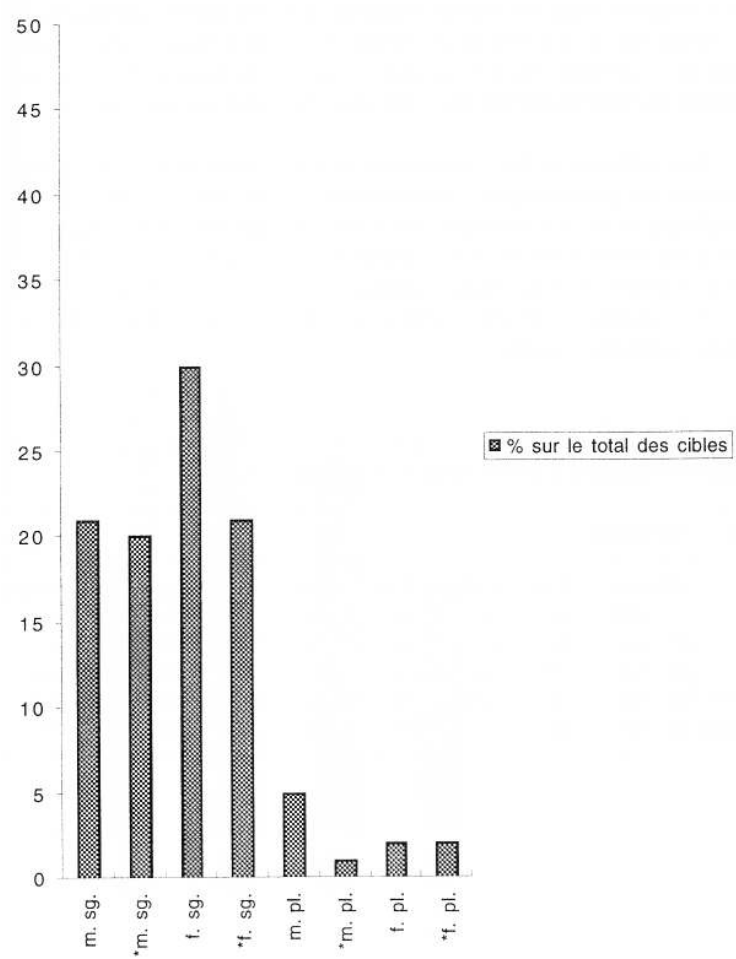

Pas plus que les autres adultes persanophones (Chini, 1992b) la mère MB n'apprend l'accord en $G$ (seules $54 \%$ des cibles sont accordées correctement); elle utilise et 
généralise presque uniquement des formes du m. (sg. et pl.). Par contre son enfant F1B emploie souvent aussi des f. sg. et pl. corrects; ses quelques erreurs (17\%) montrent surtout la généralisation $\mathrm{du} \mathrm{m}$. On trouve aussi les mêmes tendances chez les autres apprenants, sauf chez Anna, qui a, d'abord et globalement, un plus haut pourcentage de formes au f., correctes ou non, et, pour ce qui est du pluriel, chez Henri, qui généralise souvent les formes du f. ( $8 \%$ des cibles non correctes au f. pl.).

Les tableaux et les graphiques suivants montrent, pour quelques apprenants, les pourcentages d'accord correct sur le total des cibles (Tableau 6, Graphique 4) et aux moments des différents entretiens (Graphiques $5,6,7$; on a mis entre parenthèses les données basées sur peu de contextes); le Tableau 7 donne les pourcentages globaux d'accords corrects au f. sg. et pl. dans trois secteurs: articles (définis et indéfinis), adjectifs épithètes et attributs / participes passés.

Tableau 6 : Pourcentages globaux d'accords corrects sur les cibles, considérés séparément et ensemble (données orales)

\begin{tabular}{|l|l|l|l|l|l|l|l|l|l|l|}
\hline Cibles & apprenants & & & & & & & & & \\
\hline & MB & F1A & F1B & F2B & F2A & Ava & Edg. & Anna & Sar. & Henri \\
\hline déf. & - & $(50)$ & 90 & 76 & 80 & 73 & 71 & 71 & 78 & 78 \\
\hline indéf. & 57 & 66 & 81 & 85 & 65 & 75 & 78 & 57 & 62 & 92 \\
\hline épit.. & 45 & 44 & $(75)$ & 68 & 74 & 53 & 57 & 48 & 41 & 64 \\
\hline attr./PP & 36 & 58 & $(63)$ & $(45)$ & 50 & 33 & 82 & 28 & $(57)$ & 57 \\
\hline ensemble & 54 & 59 & 82 & 73 & 71 & 62 & 69 & 55 & 63 & 77 \\
\hline
\end{tabular}


Graphique 4 : Accords en G corrects d'Anna (production orale)

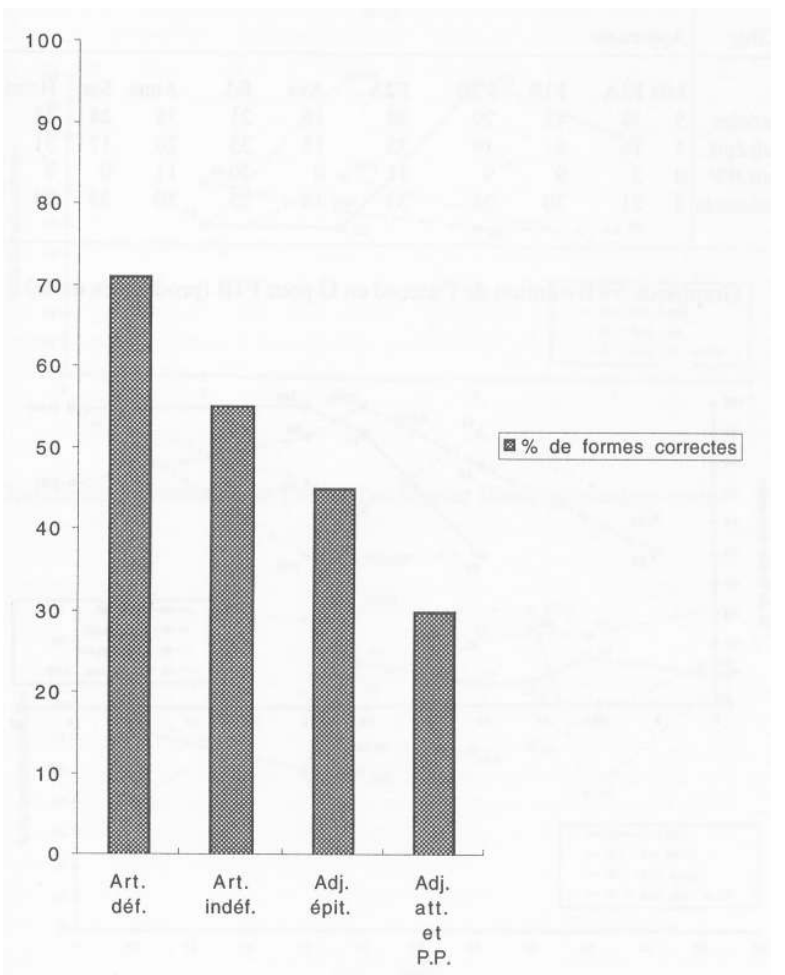

Tableau 7 : Pourcentages globaux des accords corrects au f. sg. et pl.

\begin{tabular}{|l|l|l|l|l|l|l|l|l|l|l|}
\hline Cible & Apprenant & & & & & & & & & \\
\hline & MB & F1A & F1B & F2B & F2A & Ava & Ed. & Anna & Sar. & Henri \\
\hline articles & 5 & 38 & 43 & 29 & 38 & 16 & 21 & 38 & 28 & 38 \\
\hline adj.épit. & 3 & 16 & 9 & 19 & 35 & 15 & 33 & 20 & 17 & 31 \\
\hline attr./PP & 0 & 3 & 9 & 9 & 11 & 0 & 20 & 11 & 0 & 8 \\
\hline ensemble & 3 & 21 & 30 & 24 & 33 & 14 & 25 & 30 & 25 & 34 \\
\hline
\end{tabular}


Graphique 5 : Evolution de l'accord en G pour F1B (production orale)

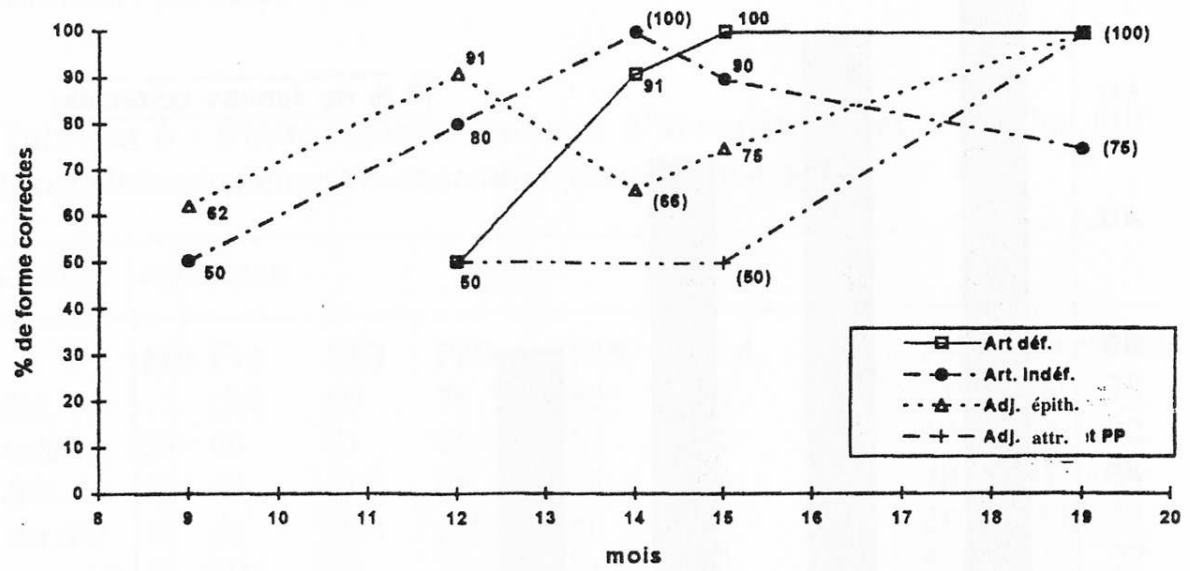

Graphique 6 : Evolution de l'accord en G pour Anna (production orale)

Anna

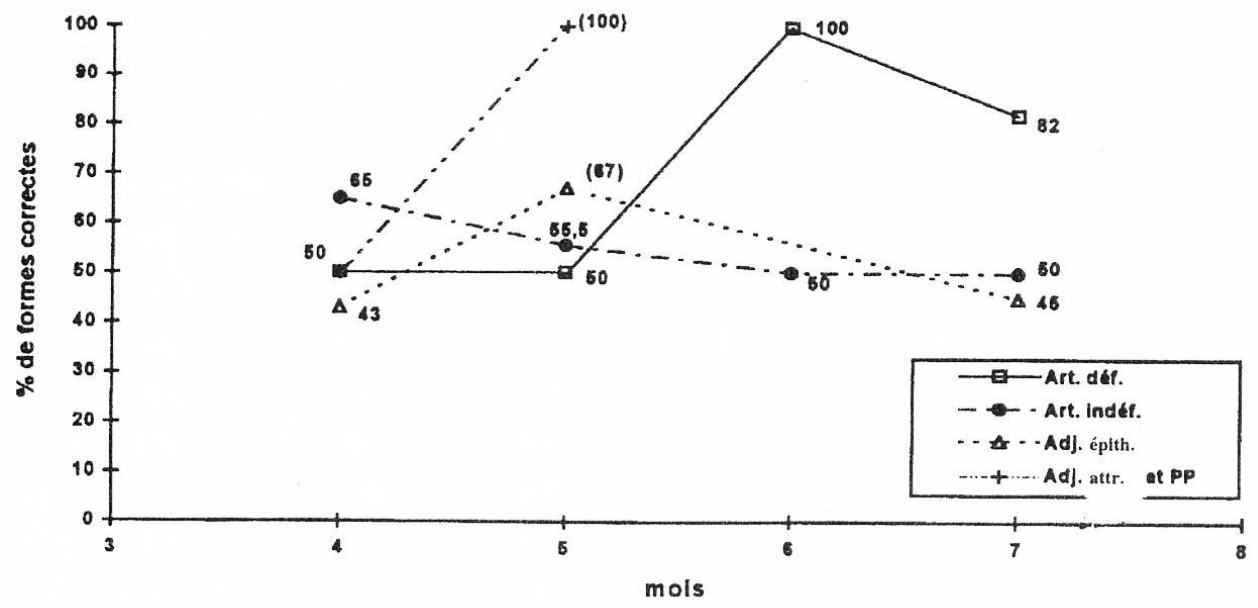




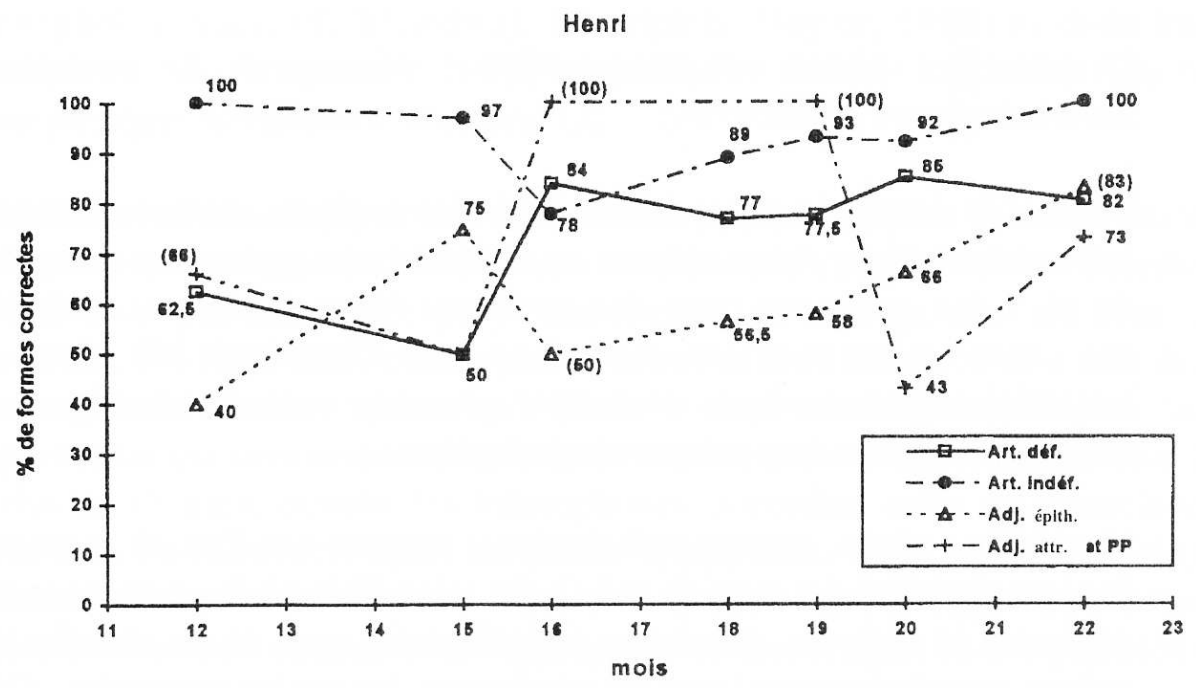

Ces données, globales et longitudinales, nous semblent confirmer la séquence acquisitionnelle proposée au début du paragraphe : les pourcentages d'accords corrects (globaux et pendant les différents moments du parcours acquisitionnel) sont en principe plus hauts dans le cas des articles, plus bas dans le cas des adjectifs épithètes et surtout dans le cas des attributs et des participes passés. Les accords corrects au féminin montrent que dans nos ILs il est plus facile de trouver au f. un article, plutôt qu'un adjectif épithète ou qu'un attribut ou un participe passé. Les apprenants moins dynamiques et moins corrects (MB, F1A, Ava, Anna, Sarah) produisent peu d'épithètes féminins corrects et presqu'aucun attribut féminin correct (cf. Tableau 6 et 7).

\section{Discussion}

L'élaboration de la catégorie du G dans l'IL est un processus long et complexe, qui touche à plusieurs niveaux de la compétence linguistique et s'appuie autant sur la découverte de principes spécifiques de l'italien (1) que sur des tendances plus générales (2-4), parfois sur des traits de la LM (5).

(1) On a pu relever quatre phases acquisitionnelles qui jalonnent la découverte de la grammaire du G dans la LC :

a - la première phase est pragmatique, dépourvue de marques morphologiques productives;

$\mathrm{b}$ - pendant la deuxième phase (plutôt lexicale) quelques marques lourdes apparaissent (p.ex. des numéraux ou des indéfinis, au lieu des marques du pl. : due settimana pour due settimane (deux semaines), MA 15 mo.; tanti estoria pour tante storie (beaucoup d'histoires), MB 15 mo. ; les articles sont employés seulement pour leur valeur sémantique et textuelle, non pour leur valeur morphologique ;

c - la troisième phase, protomorphologique, suppose que l'on ait reconnu la segmentabilité des mots de la LC. On saisit d'abord des terminaisons saillantes et typiques, au niveau phonologique, puis on en cherche la valeur morphologique, tout en employant les moins marquées. On assiste parfois à un conflit entre le caractère marqué du masculin au niveau phonologique (cf. les articles $\mathrm{m}$. un, il, avec finale consonantique) et son caractère non marqué au niveau morphologique (cf. $§ 1)$. Ce 
conflit peut être résolu en faveur du critère phonologique dans un premier temps (généralisation de la ou una, le), en faveur du critère morphologique ensuite (généralisations de un, $i l,-o$ ), plus conforme à la LC. Les apprenants semblent guidés par les régularités formelles principales de la LC concernant les classes nominales (Tableaux 1 et 2, par. 2) et les formes associées (articles, adjectifs, etc., par. 3) ;

d - enfin (phase morphosyntaxique) les marques correctes apparaissent dans les contextes d'accord les plus importants du point de vue sémantique (accord du pronom), les plus fréquents (articles), les plus favorables du point de vue de la structure linguistique (distance syntaxique réduite). Il est possible que l'utilisation fréquente de l'article, pour sa valeur sémantique et fonctionnelle (référence définie ou indéfinie, valeur anaphorique ou cataphorique), entraîne l'acquisition de sa valeur d'indice de $G$ (Wegener, $1992: 364$ ), qui reste secondaire (Pfaff, 1984).

Des stades semblables ont été signalés dans les travaux ESF sur la temporalité (stratégies pragmatiques de la variété de base $\rightarrow$ lexicales $\rightarrow$ morphologiques, cf. Bhardwaj, Dietrich \& Noyau, 1988) et dans une recherche de Wegener (1992) concernant le développement de la morphologie nominale en allemand LC.

(2) Dans nos données (comme cela arrive très probablement chez les natifs, cf. les dernières expériences de Bates et al., 1994) l'acquisition de l'accord en $G$ n'est pas influencée par la sémantique du nom (si ce n'est pour le pronom, cf. (3)), mais plutôt par sa terminaison. En outre elle suit des principes de distance syntaxique (entre le nom et ses cibles). Même des apprenants qui connaissent dans leur LM un système d'accord semblable à celui de l'italien, comme les francophones, accordent assez tôt l'article et très tard l'attribut (cf. aussi les autres apprenants, Chini, 1992a, 1994a). Nous avons donc postulé une hiérarchie d'accord syntaxique, se reflétant dans la séquence acquisitionnelle proposée ci-dessus (par. 3). Le paramètre de la distance syntaxique qui l'explique s'est déjà avéré important à propos d'études typologiques sur l'accord (Corbett, 1979 ; 1991), qui ont démontré qu'en cas de conflit entre $G$ grammatical et sexe du référent (ex. allemand das Mädchen neutre, mais se rapportant à une fille), si la distance entre le nom et la cible de l'accord est réduite, l'accord syntaxique est de plus en plus probable (alors que l'accord sémantique l'est de moins en moins) ${ }^{17}$. Cette corrélation entre données acquisitionnelles et données typologiques nous semble intéressante; elle se base vraisemblablement sur des mécanismes psycholinguistiques d'élaboration et de production des unités syntaxiques (telles que les syntagmes nominaux), mécanismes qui se reflètent dans la tendance à signaler formellement l'appartenance des cibles aux syntagmes, notamment par le moyen de l'accord grammatical.

41 (3) Quant à l'accord des pronoms personnels de 3ème personne (du sg.), il ne semble créer aucun problème, grâce à son évidente valeur sémantique et fonctionnelle (Chini, 1992a, 1994a), qui nous amène à le distinguer des autres accords, plus formels et moins «utiles » à la communication. En effet beaucoup de spécialistes (Martinet, 1956, Greenberg, 1978) considèrent les pronoms, surtout démonstratifs, comme la source diachronique du G; le caractère fondamental de l'opposition de $G$ dans le secteur pronominal, qui vaut pour un nombre élévé de langues, est formulé aussi par une généralisation (numéro 43) de Greenberg (1963): "Une langue qui possède des catégories de genre dans le nom en a aussi dans le pronom ».

(4) D'autres tendances interlinguistiques se trouvent confirmées par nos données acquisitionnelles. L'acquisition de la dérivation (et de la flexion) de $\mathrm{G}$ semble suivre des 
principes de Morphologie Naturelle (Dressler et al., 1987), selon lesquels une codification grammaticale transparente et uniforme favorise l'acquisition (cf. l'association du $\mathrm{G}$ féminin aux marques - $a$ et -essa, d'où les erreurs, de dérivation ou de flexion : direttora pour direttrice, atletessa pour atleta f., la problema, pour il problema $)^{18}$. En outre, même en tenant compte de la difficulté qu'il y a à distinguer l'expression formelle des catégories $\mathrm{du} G$ et du nombre en italien, on a constaté une certaine primauté du nombre sur le $G$ dans l'acquisition de L2 (plusieurs apprenants commencent p.ex. à accorder l'adjectif en nombre, puis en $G$; Chini, 1992b) ; cela est conforme à la généralisation 36 de Greenberg (1963), selon laquelle le nombre est une catégorie plus répandue que le G. Enfin, selon les prédictions de la théorie des marques (markedness), les formes les plus employées et généralisées sont celles du masculin singulier, les moins marquées dans la morphologie de l'italien.

(5) L'élaboration de la catégorie du G n'ignore pas le recours à la LM comme (res)source d'hypothèses grammaticales et lexicales: si la LM et la LC sont typologiquement proches (Giacalone Ramat, 1994) et si les deux systèmes en contact sont comparables quant à l'importance de la catégorie du G, de son accord et, peut-être, aux critères d'attribution du G, l'appel à la LM peut favoriser l'association des différentes désinences du domaine nominal de la $\mathrm{LC}$ à une catégorie grammaticale déjà connue, telle que le $\mathrm{G}$. L'apprentissage du $\mathrm{G}$ est donc plus rapide et pose moins de problèmes dans l'ordre aux francophones, aux germanophones, aux anglophones et enfin aux persanophones ${ }^{19}$. Parfois, surtout chez des apprenants ayant une LM proche de la LC (français, éventuellement allemand), la LM sert aussi de réservoir lexical, d'où l'on tire des lexèmes, qui sont tout de même adaptés à la morphophonologie de la LC au moyen de morphèmes de $\mathrm{G}$ cohérents (tampeta, $\mathrm{f}$. pour tempesta, d'un francophone). D'autres erreurs trahissent le recours à la LM (la mare; généralisation de l'article le au pl., chez un francophone). En général nos données sont conformes à la Markedness Differential Hypothesis (hypothèse du différentiel de marque) de Eckman (1977), selon laquelle ce sont les secteurs de la LC différents de la LM et plus marqués (p. ex. accord de l'attribut) qui créent plus de difficultés aux apprenants ${ }^{20}$.

\section{Conclusion et comparaison}

En conclusion, l'abondance des marques de flexion dans le syntagme nominal italien semble pousser l'apprenant à développer assez vite (quoique graduellement) une sensibilité morphologique et formelle, surtout si sa LM (comme dans le cas du français ou de l'allemand) l'oriente dans cette direction. Cette sensibilité a déjà été observée pour le secteur verbal de l'italien L2 par Giacalone Ramat (1993a), qui, du moins pour ce secteur, souligne que les apprenants d'italien LC diffèrent des apprenants d'allemand, français ou anglais, langues où la morphologie est moins évidente et transparente. La dite sensibilité morphologique, notamment dans le domaine $d u G$, est beaucoup plus évidente chez les enfants italophones, dont l'acquisition du $\mathrm{G}$ procède très vite, presque sans fautes, à part quelques régularisations semblables à celles des apprenants adultes (accord f. après des noms m. en - $a$; Chini, 1992a, 1994a).

Comment peut-on expliquer ces analogies et ces quelques différences? Le comportement d'enfants natifs et d'apprenants adultes quant à l'acquisition du $G$ est souvent comparable parce qu'il se fonde sur des indices (cues) disponibles et fréquents (essentiellement des associations entre une terminaison nominale et un $\mathrm{G}$, des facteurs 
phonologiques, cf. Bates et al., 1994), auxquels même les réseaux connectionistes sont sensibles (MacWhinney et al., 1989 pour le G en allemand, Sokolik-Smith, 1992 pour le français). Mais des différences existent néanmoins, car chez les apprenants, enfants ou adultes, d'autres éléments jouent eux aussi un rôle (Chini, 1994a). Il nous semble que chez les enfants la catégorisation formelle du $\mathrm{G}$ est d'une part cohérente avec le type flexionnel de la LM, et, d'autre part, qu'elle s'accompagne au développement de la catégorisation conceptuelle et de l'identité sexuelle, qui ont une importance affective et cognitive assez profonde. Par contre chez les adultes le $G$ est presque uniquement un phénomène formel, dont la fonction est réduite et que l'on apprend quand il est sémantiquement important ( $\mathrm{G}$ du pronom se rapportant à des animés sexués, cf. Andersen, 1984b) et/ou si sa codification est transparente, envahissante, comparable en quelque sorte à celle de la LM. Somme toute, si une explication fonctionnelle est nécessaire d'après nos données, à la différence des conclusions de Wegener (1992) concernant l'allemand LC, nous estimons que celle-ci n'est pas suffisante pour le G en italien LC et qu'il faut considérer autant le rôle de la typologie des langues en contact qu'une remarquable sensibilité des apprenants aux facteurs formels les plus transparents.

\section{NOTES}

1. On utilisera donc les abréviations suivantes : $\mathrm{LM}=$ langue maternelle $; \mathrm{LC}=$ langue cible $; \mathrm{IL}=$ interlangue $; \mathrm{G}=$ genre $; \mathrm{m} .=$ masculin $; \mathrm{f}_{\text {. }}=$ féminin $;$ sg. = singulier $;$ pl. = pluriel $;$ mo. = mois.

2. C'est un domaine qui n'a pas été beaucoup étudié jusqu'à présent, étant donné que la recherche italienne s'est concentrée surtout sur le verbe et sur les catégories de la temporalité et de la modalité (Bernini \& Giacalone Ramat, 1990 ; Giacalone Ramat, 1992, 1993a et 1993b ; Banfi, 1993 ; Giacalone Ramat \& Crocco Galèas, 1995). Quant aux travaux sur le domaine nominal des interlangues de l'italien, ils ont privilégié une approche morphologique (Berretta, 1986, 1990 ; Chini, 1992a, 1992b, 1994a ; Valentini, 1990 ; Berretta \& Crotta, 1991), sans oublier quelques considérations fonctionnelles et notionnelles (cf. Berruto et alii, 1990). La fonction discursive et référentielle du syntagme nominal dans les interlangues est par contre peu connue pour le moment (mais cf. Valentini, 1992).

3. Dans ce cas-là, comme il arrive en français, c'est le pronom clitique objet préverbal qui régit l'accord: le f. pl. ho mess-e là (Je les ai mis-es là-bas) (cf. Marcantonio \& Pretto, 1988 ; Serianni, 1988).

4. Mais il y a des exceptions, comme soprano, de $\mathrm{G}$ m. et se rapportant normalement à une femme ; guida (guide) de $\mathrm{G}$ f. employé tant pour des hommes que pour des femmes.

5. Là où les deux pluriels sont possibles, ils ont des connotations sémantiques différentes: le masculin a une valeur singularisante (muri [murs]), tandis que le féminin est collectif (mura [murs, murailles]) ou se rapporte à des parties du corps (braccia f. [bras (humains)]) vs. bracci m. [bras (d'un fleuve, p.ex.)].

6. La différence peut n'être que graphique (cf. dur m. vs. dure f.), à moins que le morphème -e du f. ne cause la conservation de la consonne finale (grand vs. grande) ou que d'autres phénomènes n'interviennent (vif vs. vive, sec vs. sèche). 
7. D’autres apprenants ont été suivis plus épisodiquement (p. ex. Ken, francophone du Zaïre). On se concentrera ici sur les données recueillies à des intervalles réguliers d'un mois auprès des treize apprenants mentionnés, en enregistrant des conversations semi-libres (de 20' à 40') qui portaient sur des expériences personnelles et des sujets quotidiens, des descriptions, des reformulations de récits. Ces enregistrements étaient suivis d'un court test écrit concernant la morphologie (cf. Chini, 1992a ; 1992b ; 1994a et 1994b).

8. Les quelques fautes de terminaison se trouvent surtout chez des persanophones adultes (les enfants sont beaucoup plus corrects : Chini, 1992a) et des anglophones, moins souvent chez des germanophones (Chini, 1994a), p. ex. pantalina pour pantaloni / e m. (pantalon) (MA) ; faccio pour faccia f. (face) (MB, PB); zuppo pour zuppa f. (soupe) (Ava), polvero pour polvere f. (poussière, poudre) (Sarah)

9. D'autres exemples de féminins non conformes à la LC, mais révélateurs des tendances des ILs, sont : cantanta pour cantante (hypercaractérisation), lea pour leonessa, canessa pour cagna, atletessa pour atleta (m. et $\mathrm{f}$.), enfin deux (pseudo-) diminutifs, orsetta pour orsa, infermierina pour infermiera (tous chez Henri, 20 mo.). Chez un sinophone, Valentini $(1990: 342)$ trouve surtout des formes f. en - $a$ : professosa pour professoressa, ministra, sindaca.

10. Pour l'italien on a relevé l'application de ces principes dans les ILs (Bozzone Costa, 1986, 1994 ; Berretta, 1987) et dans les variétés d'enfants italophones (Lo Duca, 1990).

11. Traduction : J'ai vu Ø lunapark + puis + dans Ø lunapark il y a (le) cirque + puis allé dedans + acheté $\varnothing$ bi/ billet [...] après beaucoup de gens + avec cela + eh il y a $\varnothing$ bicyclette qui avec une roue + après (ils) allaient sur ce bicyclette + après (ils) font une avion.

12. Il y a des généralisations de formes du m. (tanti gente pour tanta gente, f. sg.; quello bicicletta au lieu de quella bicicletta $\mathrm{f}$.). Quant aux articles, on observe un emploi préférentiel de morphèmes $\mathrm{f}$. (una ruota, correct ; una aereo, pour un aereo, un nom en -o, typiquement m.).

13. On a utilisé les parenthèses là où la séquence est assez probable, sans être tout à fait certaine. Les graphiques et les tableaux à la fin du paragraphe illustreront cette séquence.

14. L'emploi des clitiques est plus tardif et suit la séquence $: l o>l a>l i>l e$, conforme au caractère non marqué du m. et du sg. (Chini, 1992a et 1994a pour l'italien L1 et L2 ; Berretta, 1986).

15. Il faut considérer qu'en persan il n'y a pas d'article défini correspondant à celui de l'italien ; on utilise seulement un suffixe (-r,) pour les objets définis : Hasan-r, didam (j'ai vu Hassan) (Chini, 1992b : 451-452; Windfuhr, 1979).

16. Cf. mia sorella (ma sœur), F1A, 10 mo ; la mia sciarpa (mon écharpe), F2A, 15 mo., tutta la notta (toute la nuit) Ava, 9 mo. (avec notta pour notte f. hypercaractérisé), tous accordés ; mais un piccolo sauna (un petit sauna), Anna 7 mo., molto acqua (beaucoup d'eau), Henri 19 mo., della televisione italiano (de la télévision italienne), Henri, 12 mo., non accordés.

17. La hiérarchie formulée par Corbett $(1991: 226)$ est la suivante : épithète > attribut > pronom relatif > pronom personnel. La probabilité d'un accord grammatical diminue et la probabilité d'un accord sémantique augmentent de gauche à droite : avec un pronom personnel il est plus fréquent de trouver un accord sémantique qu'avec un prédicatif (ex. : le soprano est très connu (m.) ; il m. / elle f. a chanté dans les plus importants théâtres du monde).

18. Il s'agit d'erreurs de genre différent, mais qui trahissent les mêmes principes. Dans le premier cas on remplace un morphème peu fréquent (-trice) par un morphème plus fréquent (a) ; dans le deuxième une marque typique, mais faible, $-a$, par une plus lourde, -ess $a$; dans le troisième la terminaison du nom - $a$ donne lieu à un accord de l'article au f., incorrect mais cohérent.

19. Le rôle de la LM émerge aussi d'après les données de Pfaff (1984) et Wegener (1992) : les apprenants ayant comme LM le turc (sans $G$ ) apprennent le $G$ de l'allemand LC plus tard respectivement que les grecs et les slaves. 
20. On néglige ici d'autres facteurs communicatifs, socioculturels, personnels, tels que l'âge, le style cognitif et la motivation, qui peuvent accélérer ou ralentir l'acquisition du G (cf. Chini, 1992a, 1992b).

\section{RÉSUMÉS}

Cet article porte sur l'acquisition d'une catégorie fondamentale pour la grammaire du nom et du SN de l'italien: le genre grammatical. Après une description du genre de l'italien comme phénomène lexical et flexionnel ayant plusieurs effets syntaxiques, cette étude présente et compare les données acquisitionnelles de quatre groupes d'apprenants de différentes langues maternelles (persan, anglais, allemand, français). Il en ressort que ces apprenants sont sensibles aux propriétés formelles et sémantiques saillantes du genre italien et que, malgré une influence claire de leur L1, ils suivent tous la même séquence d'acquisition de l'accord, séquence qui est cohérente avec des principes identifiés par la typologie linguistiques et par quelques études sur d'autres L2.

This paper deals with the acquisition of a category which is very pervasive in Italian NP and nominal grammar : grammatical gender. After a short description of Italian gender as a lexical and an inflectional phenomenon with syntactic implications, the study presents and compares the acquisitional data of four groups of learners having different L1s (Persian, English, French and German). The results indicate that these learners are sensitive to the main formal and semantic properties of Italian gender and that, in spite of a clear influence from the L1, they all show the same acquisitional sequence for gender agreement, which is consistent with some semantic and syntactic principles observed by typological linguistics and by a few acquisitional studies on other L2.

\section{AUTEUR}

\section{MARINA CHINI}

Université de Pavie 